\title{
El consumo de nitrato y su potencial efecto benéfico sobre la salud cardiovascular
}

\author{
Nitrate consumption and potential \\ beneficial effect on cardiovascular health
}

\begin{abstract}
Vegetable consumption is associated with a lower risk of suffering cardiovascular disease and cancer, traditionally ascribed to their content in antioxidants. Recently it has been proposed that this effect may be due to the presence of nitrates in vegetables. Cardiovascular diseases are usually associated with an impaired production of nitric oxide (NO) in blood vessels. One strategy aimed to correct this defective production is to generate NO from inorganic nitrates contained in vegetables. However, it has been traditionally thought that nitrites and nitrites, as those used in curing meats and as food preservers, could generate adducts that may increase cancer risk. This paradigm is now being revisited since the evidence that vegetables rich in nitrates may have a beneficial impact on human health, particularly on cardiovascular parameters, has showed promising results, although more complete clinical evidence is needed.
\end{abstract}

Key words: nitrite, nitrate, nitric oxide, arterial hypertension, nitrosilation.
Brayan Moreno C. (1)

Karen Soto O. (1)

Daniel González R. (1,2)

(1) Departamento de Ciencias Básicas Biomédicas, Facultad de Ciencias de la Salud, Universidad de Talca, Talca, Chile. 2) Programa de Investigación de Excelencia Interdisciplinario en Envejecimiento Saludable (PIEI-ES), Universidad de Talca, Talca, Chile.

$$
\begin{array}{r}
\text { Dirigir la correspondencia a: } \\
\text { Dr. } \\
\text { Daniel Gonzalez Reinoso } \\
\text { Departamento de Ciencias Básicas Biomédicas } \\
\text { Facultad de Ciencias de la Salud } \\
\text { Universidad de Talca } \\
\text { Avenida Lircay s/n } \\
\text { Talca, Chile } \\
\text { Fono 56-71-2-418856 } \\
\text { E-mail: dagonzalez@utalca.cl }
\end{array}
$$

Este trabajo fue recibido el 10 de Septiembre de 2013, aceptado con modificaciones el 15 de Mayo de 2014 y aceptado para ser publicado el 30 de Enero de 2015.

para el tratamiento de las enfermedades cardiovasculares.

Varios ensayos clínicos han mostrado que una dieta rica

Desde los años 80 se identifica al oxido nítrico (NO), como el "factor de relajación derivado del endotelio" (1), dando paso a la investigación continua de su síntesis, función y regulación. EI NO es un gas lipofílico con capacidad de atravesar las membranas celulares. En su estructura química presenta un electrón libre o no apareado, situación que le entrega la capacidad de reaccionar con múltiples moléculas. Su producción es parte de un proceso enzimático catalizado por la enzima óxido nítrico sintasa (NOS), en la cual el aminoácido L-arginina es oxidado a L-citrulina con la consecuente producción de NO.

En el sistema cardiovascular, el NO regula varios procesos, como el tono vascular $(2)$, la función cardíaca $(3,4)$, la agregación plaquetaria (5) y la permeabilidad vascular $(6,7)$. Estos efectos inducidos por el NO son mediados a nivel celular por dos mecanismos principales: la generación del segundo mensajero guanosina monofosfato cíclico $3^{` 5}$ ` (GMPc) a partir de guanosina trifosfato (GTP) por activación de la enzima guanilato ciclasa, y por acción directa del NO sobre proteínas a través de mecanismos redox (2-4).

En general, las enfermedades cardiovasculares se asocian a una disminución de la producción de $\mathrm{NO}$ a través de la ruta de la L-arginina $(2,4,8-10)$. De allí que la restauración de la producción de NO aparece como una estrategia interesante en frutas y verduras reduce la presión arterial y los eventos cardiovasculares (11,12). Algunos vegetales como la lechuga o la espinaca tienen un alto contenido de nitrato inorgánico, por lo que es posible que el contenido de estos iones contribuya a ejercer parte de los efectos benéficos de esta dieta. Sin embargo, los nitratos contenidos en los alimentos han sido considerados potencialmente peligrosos para la salud. Se ha sugerido su asociación con el cáncer, particularmente de estómago y colon (13). Esto deriva de la formación endógena de $\mathrm{N}$-nitroso-compuestos, los que podrían tener efectos saminas y nitrosamidas (15). Existen evidencias de que el alto consumo de embutidos y otros tipos de alimentos procesados que poseen gran cantidad de nitratos tienen efectos dañinos para la salud. Esto se puede apreciar en el estudio de Mirvish et al (16), quienes evaluaron el efecto de una dieta rica en embutidos sobre el colon de ratones. Sus resultados apoyan la hipótesis de que la formación de N-nitroso-compuestos a partir de alimentos ricos en nitratos, serían una causa del cáncer de colon. Sin embargo, pese a la intensa investigación en el área, no se ha podido demostrar una directa participación del nitrato o nitrito en el proceso de carcinogénesis (17). cancerígenos (14). Ejemplo de estos compuestos son las nitro- 
En los humanos, tras la absorción en la pared estomacal, alrededor de un $25 \%$ de los nitratos consumidos entran en la circulación entero-salival, donde son reducidos a nitritos por acción de la nitrato reductasa de bacterias anaerobias facultativas de la superficie dorsal de la lengua (18). Este nitrito naciente es tragado y reducido a $\mathrm{NO}$ por el $\mathrm{pH}$ ácido del estómago, o re-ingresa a la circulación como nitrito (19). Aparte de la vía entero-salival, Cosby et al. (20) establece que el nitrito puede ser transformado en NO por acción de la deoxihemoglobina en condiciones de hipoxia y por la enzima xantino oxidoreductasa (21).

El nitrito ha mostrado tener un rol protector en el daño producido por isquemia-reperfusión en el miocardio (22), el hígado (23) riñón $(24)$ y cerebro $(25,26)$. Con estos antecedentes, es posible pensar que los nitratos contenidos en los vegetales tengan un rol cardioprotector.

\section{NITRATOS, NITRITOS Y SU RELACIÓN CON EL ORGANISMO}

Los nitratos son compuestos que están presentes en el medio ambiente en forma natural como consecuencia del ciclo del nitrógeno. La distribución de estos iones es muy variada, encontrándose en suelos, alimentos y aguas. Por este motivo, cualquier modificación antropogénica del ciclo natural del nitrógeno cambiará las concentraciones normales de nitrato y nitrito en el ambiente. El nuevo paradigma acerca del consumo de nitratos y nitritos cuestiona la visión del nitrato como un residuo no deseado y tóxico para el organismo. Recientemente se ha propuesto que el consumo de nitrato ayudaría a mejorar diversas alteraciones metabólicas, a través del NO. Según estudios recientes (27), existiría una ruta o vía inversa en que el nitrato inorgánico actúa como sustrato para la generación de $\mathrm{NO}$ en condiciones de hipoxia, donde la enzima óxido nítrico sintasa (NOS) no realiza su función. Es decir, el NO generado por esta vía seria un complemento al que se produce por la vía endógena de la L-arginina en condiciones normales de oxigenación y con acción normal de la NOS (19).

El reconocimiento de esta ruta ha llevado a varios grupos de investigación a explorar el papel de los nitratos y nitritos en los procesos fisiológicos reconocidamente regulados por el NO. Más aún, este principio ha llevado a la hipótesis que el nitrato participa en la vasodilatación producida por la hipoxia y en la regulación del consumo de oxígeno a nivel mitocondrial. También se predice un papel citoprotector, en el contexto de la isquemia y reperfusión patológica (23).

\section{Ingreso de los nitratos al organismo}

Estos iones poseen diferentes formas de ingreso al organismo. La vía entero-salival es clave para el nitrato inorgánico ingerido por vía oral. Las verduras de la dieta son la fuente principal, ya que representan el $60-70 \%$ de la ingesta de nitratos al día en personas con una dieta del tipo occidental (28). Una vez que se ingiere, el nitrato se absorbe rápidamente a nivel gastrointestinal y se mezcla con el nitrato sintetizado endógenamente, proveniente principalmente de la oxidación del NO (29). La mayor parte del nitrato en última instancia se excreta por la orina.

Aproximadamente un cuarto del nitrato del plasma es absorbido por las glándulas salivales y secretado en la saliva, alcanzando concentraciones al menos 10 veces superiores a las concentraciones plasmáticas (30-32). El nitrato salival se reduce a nitrito por acción de bacterias comensales de la cavidad oral. Esto ocurre gracias a la existencia de la enzima nitrato reductasa en estos microorganismos (33). Se han obs- rvado por lo menos tres tipos de esta enzima. Algunas de las bacterias que cuentan con las tres isoformas son las del genero paracoccus, Escherichia coli y la Salmonella typhimurium (34).

Por otra parte, en el ambiente ácido estomacal, el nitrito salival es reducido a NO y a otros compuestos derivados del nitrógeno, que pudiesen tener posibles efectos cancerígenos (35). Sin embargo, a pesar de los extensos estudios en este ámbito, la relación de las bacterias comensales, nitrato y el cáncer gástrico no está clara (17).

Mecanismos de reducción de los nitritos en el organismo

Una vez que se forma el nitrito, existen varias vías en el organismo para su reducción a NO (3), como son a través de la acción de la hemoglobina, mioglobina, algunos antioxidantes como el ascorbato, polifenoles y protones (36). La generación de NO por estas vías es mucho mayor durante la hipoxia y la acidosis, lo que garantiza la producción de NO en situaciones en las que las actividad oxígeno- dependiente de la NOS está comprometida (9).

La hemoglobina se comporta como una nitrato reductasa regulada alostericamente. Según Cosby et al. (20) durante la infusión de nitrito en la circulación sanguínea, los efectos vasodilatadores están asociados con la formación de NO, medido por la formación de NO unido al grupo hemo de la hemoglobina. Se ha descrito que varias sistemas enzimáticos, incluyendo la xantina oxidorreductasa, los complejos de la cadena transportadora de electrones mitocondrial (37), el citocromo P450 y la NOS, podrían utilizar el nitrito como un aceptor de electrones alternativo al oxígeno molecular, formando de este modo NO (36). Para cumplir un posible papel en la vasorregulación y señalización, estas vías requieren de bajas tensiones de oxígeno para generar NO de manera efectiva (38).

\section{NITRATOS, NITRITOS Y SU RELACIÓN CON EL CÁNCER}

La presencia de nitratos en alimentos y en aguas podría generar la formación endógena de $\mathrm{N}$-nitroso-compuestos con posibles efectos carcinógenos (35). La relación entre nitratos, nitritos y el cáncer gástrico se ha sugerido por la generación de N-nitroso-compuestos a partir de aminas secundarias, especialmente por el consumo de carnes procesadas, a las que se les agrega nitrito de sodio para su preservación (39). Desde el inicio de estas investigaciones, alrededor del 90\% de los compuestos del grupo nitrosamina estudiados se consideraron como cancerígenos, ya que producían tumores hepáticos en animales de experimentación (15). Estos compuestos derivados del nitrógeno se forman por procesos de nitrosación. En condiciones de $\mathrm{pH}$ bajo y en presencia de aminas, los nitratos consumidos en la dieta pueden formar nitrosaminas. Este proceso podría explicar el vínculo existente entre los productos que contienen nitratos y la nitrosación a nivel del tubo digestivo (40).

Se ha mencionado que las nitrosaminas pueden ocasionar cáncer en animales y en humanos. Hasta el momento, las evidencias epidemiológicas demuestran una cierta correlación entre la ingesta de nitratos y el riesgo de cáncer gástrico $(40,41)$. Por otra parte, estudios realizados en regiones europeas muestran lo contrario, que no existe correlación entre una dieta rica en nitrato y cáncer gástrico (42). Resultados similares se obtuvieron en ciertos estados norteamericanos y en países asiáticos (43). Por otra parte, las tasas de incidencia de mortalidad por cáncer de estómago se han reducido considerablemente en casi todos los países (16). Este descenso 
se podría atribuír de alguna manera a la refrigeración de los alimentos, lo que evita el agregado de aditivos que contienen altas concentraciones de nitrato.

También se ha propuesto que algunas moléculas antioxidantes como flavonoides, ácido ascórbico, polifenoles y otros, presentes en los vegetales, podrían contribuir a la disminución de las cifras de enfermedades neoplásicas, ya que estos disminuyen la formación de nitrosaminas (44). La betarraga, por ejemplo, es rica en nitratos y contiene antioxidantes como las betalinas y ácido ascórbico. Este último se ha descrito como un inhibidor de la reacción de S- nitrosilación $(45,46)$ y de la formación de nitrosaminas. De esta manera, los nitratos contenidos en los vegetales podrían generar NO sin producir compuestos cancerígenos (47).

En resumen, pese a la intensa investigación en el área, no se ha podido demostrar una directa participación de los nitratos y nitritos en la incidencia del cáncer (48). Aun más, estos iones han mostrado un papel gastroprotector. En un modelo de gastritis, el nitrato redujo el daño ulcerativo (49;50). Además, el nitrito aumentó el flujo sanguíneo hacia la mucosa gástrica y el grosor del mucus secretado (51), efecto que se sabe es mediado por NO (52). En otras palabras, los nitratos contenidos en vegetales o administrados directamente favorecen el flujo sanguíneo hacia el estómago, protegiéndolo de sustancias irritantes. Dados estos antecedentes, es probable que los nitratos contenidos en vegetales ricos en moléculas antioxidantes como el ácido ascórbico, no produzcan aductos cancerígenos, a diferencia de los contenidos en las carnes rojas.

\section{NITRATOS Y NITRITOS EN LOS VEGETALES}

El nitrato es parte integral del ciclo del nitrógeno en el medio ambiente. Este se forma a partir de fertilizantes, plantas en descomposición, residuos orgánicos, estiércol y otros. Los seres vivos pueden llegar a hacer uso de este nitrógeno a través de un proceso que se inicia con la fijación de este en los suelos, proceso realizado por los vegetales y por algunas bacterias nitrificantes asociadas (53). Las plantas captan el nitrógeno por absorción de las raíces en forma iónica, preferentemente como NO3-, y menos frecuente como NH4+. En general los nitratos se acumulan mayoritariamente en las hortalizas, y su acumulación va a depender de diversos factores como la especie, variedad y genotipo.

Según la OMS una persona consume normalmente entre 50-150 mg al día de nitrato, por ello es importante conocer la composición de los alimentos y la frecuencia con que deben consumirse. Según un estudio publicado por expertos de la European Food Safety Authority (EFSA), se debe consumir aproximadamente 400 gramos diarios de una mezcla de frutas y verduras. Esta cantidad no sobrepasaría el umbral límite de consumo de nitratos que se denomina Ingesta Diaria Admisible (IDA) recomendada por la Organización Mundial de Agricultura y Alimentos (FAO) y la OMS. Lo recomendable seria la ingesta de al menos $1 \mathrm{mmol}$ de nitrato al día para obtener efectos benéficos sobre la salud cardiovascular y evitar posibles efectos adversos (54).

Los vegetales se pueden clasificar de acuerdo a su contenido de nitrato (tabla 1). Dentro de los que presentan un mayor contenido de este compuesto destacamos algunos de interés nutricional.

\section{Betarraga}

La betarraga (Beta vulgaris L) pertenece a la familia de las chenopodiáceas. Es una planta bienal en su primer año de cultivo y produce una roseta de hojas de diferentes formas y un tubérculo de color rojizo. Nutricionalmente el aporte de esta hortaliza no es importante en vitaminas, pero provee importantes cantidades de carbohidratos. Además contiene betalinas como la betacianina (pigmento rojo) que le da su pigmentación característica (55), ácido ascórbico, retinol, y carotenoides, que le otorgan una alta capacidad antioxidante (56). El contenido de nitrato en la betarraga es de los más altos dentro de las hortalizas, con alrededor de 1800 mg NO3-/ kg masa fresca $(54 ; 57 ; 58)$, valor menor a lo establecido por algunos países europeos, en donde el máximo tolerable para la betarraga es de alrededor de $3000 \mathrm{mg} / \mathrm{kg}$ masa fresca.

Lechuga

La lechuga (Lactuca Sativa L.) es otro vegetal que posee cantidades importantes de nitrato $(54,57,58)$, que absorbe del suelo, donde una parte de estos son reducidos en la raíz y la

TABLA 1

Clasificación de los vegetales de acuerdo al contenido de nitrato ( $\mathrm{mg} / \mathrm{kg}$ de masa fresca).

\begin{tabular}{|c|c|c|c|c|}
\hline $\begin{array}{l}\text { Muy bajo } \\
<200\end{array}$ & $\begin{array}{c}\text { Bajo } \\
200-500\end{array}$ & $\begin{array}{c}\text { Medio } \\
500-1000\end{array}$ & $\begin{array}{c}\text { Alto } \\
1000-2500\end{array}$ & $\begin{array}{c}\text { Muy alto } \\
>2500\end{array}$ \\
\hline Ajo & Achicoria & & Apio nabo & Acelga \\
\hline Alcachofa & Brócoli & Nabo & Escarola & Apio \\
\hline Cebolla & Coliflor & Repollo & Perejil & Betarraga \\
\hline Esparrago & Pepino & & Puerro & Espinaca \\
\hline Melón & Zanahoria & & & Lechuga \\
\hline Papa & Zapallo & & & Rábano \\
\hline \multicolumn{5}{|l|}{ Pera } \\
\hline \multicolumn{5}{|l|}{ Sandia } \\
\hline Tomate & & & & \\
\hline
\end{tabular}


otra parte es llevada a los órganos aéreos. Se ha estimado que este vegetal cultivado en Chile posee un contenido de nitratos en un rango de 2500- $3000 \mathrm{mg} \mathrm{NO3-/kg}$ masa fresca $(59,60)$.

Rabanito

El rabanito (Raphanus sativus $\mathrm{L}$ ) también se considera un vegetal que contiene cantidades importantes de nitrato $(54 ; 57 ; 58)$. Según Paillán et al, (61), este tendría un contenido de nitrato en el rango de los 880-2560 mg/kg masa fresca, datos obtenidos en base a un estudio bajo condiciones de cultivo orgánico en invernadero. El rabanito contiene además ácido ascórbico, lo que es importante para la química del nitrato en el organismo.

\section{Espinaca}

Otro vegetal de alto contenido en nitratos es la espinaca (Spinacea Oleracea L.) (54;57;58), con hasta 4000 mg NO3-/ $\mathrm{kg}$ masa fresca. La espinaca también contiene acido ascórbico, lo que ayudaría a prevenir las reacciones de $\mathrm{N}$-nitrosación $(44,45,62)$.

\section{EFECTOS CARDIOVASCULARES DE LOS NITRATOS}

Varios estudios han mostrado que el nitrato, o dietas ricas en él, pueden inducir una reducción en la presión arterial (63). Recientemente se ha descrito que el consumo de jugo de betarragas en humanos induce un aumento en los niveles de nitratos y nitritos plasmáticos, efecto que se correlaciona con una disminución de la presión arterial, mejorando la función endotelial y disminuyendo la agregación plaquetaria (64). Además, el jugo de betarragas mostró capacidad antioxidante y anti-inflamatoria (65), efectos que han sido clásicamente atribuidos al NO.

Larsen et al, describieron una reducción en la presión arterial diastólica en voluntarios sanos a los que se les administró una dosis de nitrato de sodio de 0,1 mmol/día/ $/ \mathrm{kg}$ de peso (66). Webb et al describieron una reducción significativa de la presión sistólica en voluntarios sanos que consumieron jugo de betarragas, efecto que se correlaciona con la elevación del nitrato plasmático y que se anula por la eliminación de la saliva (64). Esto fue verificado por Coles et al en una ensayo clínico (67). Hobes et al también observaron este efecto del jugo de betarragas, de manera dosis-dependiente (68). Utilizando nitrato de potasio, Kapil et al describen una reducción dosis-dependiente de la presión arterial en voluntarios sanos, efecto que también observaron utilizando jugo betarragas como fuente de nitrato (69). Sobko et al reportaron una reducción de la presión diastólica en voluntarios sanos que consumieron nitrato a través de vegetales usados en la comida tradicional Japonesa (70). Por su parte, Bondono et al observaron una reducción de la presión sistólica en voluntarios que consumieron una dieta rica en nitratos en base a espinacas (71). Consistente con estas observaciones, Dejam et al demostraron que la infusión por vía intravenosa de nitritos es capaz de producir una vasodilatación significativa que reduce la presión arterial media en voluntarios sanos (62).

Sin embargo, otros estudios mostraron una disminución muy discreta de la presión arterial $(72,73)$ y en algunos estudios más recientes no se encontró un descenso en la presión arterial $(30,74)$.

Aunque en la mayoría de estos estudios, el número de participantes es pequeño y los voluntarios son mayoritariamente normotensos, el efecto cardiovascular del consumo de nitratos, ya sea puro o contenido en vegetales, es consistente y reproducible como lo indica un reciente meta-análisis de 16 estudios clínicos (75). En este análisis los autores concluyeron que el tratamiento con nitratos en pacientes sanos disminuye la presión arterial sistólica en aproximadamente $4,4 \mathrm{~mm}$ $\mathrm{Hg}$. Los estudios anteriormente citados fueron realizados en voluntarios sanos. En estos sujetos, el efecto vascular del nitrato probablemente sea mucho menor comparado con la producción endógena de NO. Posiblemente en sujetos con patologías como la hipertensión, con la presencia de disfunción endotelial y una menor biodisponibilidad de NO, los efectos del nitrato puedan ser más relevantes.

\section{HIPERTENSIÓN}

Los estudios anteriormente mencionados se basan, en general, en los efectos de nitratos sobre voluntarios jóvenes sanos. Recientemente concluyó el primer ensayo clínico del uso de nitratos en la dieta en una cohorte de pacientes hipertensos (ensayo clínico NCT01405898). En este estudio, el consumo por cuatro semanas de jugo de betarragas una vez al día ( 6,5 mmoles de nitrato al día) redujo la presión arterial sistólica en aproximadamente $7-8 \mathrm{~mm} \mathrm{Hg}$, comparado con un placebo (jugo de betarragas libre de nitratos). Cabe notar que estos efectos del nitrato se obtuvieron con los pacientes consumiendo su medicación anti-hipertensiva habitual (31).

Adicionalmente, un estudio en adultos mayores que presentaban un aumento moderado de la presión arterial, el tratamiento con nitrato ( $10 \mathrm{mmol}$ al día) redujo la presión arterial en aproximadamente $8 \mathrm{~mm} \mathrm{Hg}$ y mejoró la función vascular (76). En otro estudio con pacientes de la tercera edad, el tratamiento con nitratos ( $4 \mathrm{mmol} /$ día) provenientes de la dieta (betarragas, espinacas) mejoró la perfusión cerebral regional, importante en procesos cognitivos (77).

Aunque estos hallazgos son muy alentadores, quedan pendientes estudios de más largo plazo, multicéntricos y con un mayor número de pacientes (estudios de fase III). De manera notable, no se han reportado efectos adversos, aunque hay que considerar que son estudios cortos.

\section{Efectos cardiacos}

En el corazón, el NO regula varias funciones como la fuerza contráctil, la relajación del miocardio, la respiración mitocondrial y la perfusión coronaria (78), y posee claramente un rol cardioprotector en patologías como el infarto al miocardio y la insuficiencia cardiaca (2). Los blancos son variados como canales iónicos (3), enzimas de la respiración mitocondrial y moléculas involucradas en la apoptosis (4). Por ende, su manipulación farmacológica es de gran interés terapéutico. Al igual que en la vasculatura, los nitratos y nitritos inorgánicos aparecen como una alternativa interesante.

En modelos animales de isquemia-reperfusión cardíaca, el tratamiento con nitratos produjo un claro efecto cardioprotector (79-82). Sin embargo, los resultados de los estudios realizados en humanos no son tan evidentes. En un estudio con pacientes susceptibles a isquemia cardiaca, el tratamiento con nitrito de sodio mejoró los parámetros cardiacos en un protocolo de estrés inducido por dobutamina (83). Por el contrario, en un ensayo clínico muy reciente, en 229 pacientes que sufrieron un infarto agudo al miocardio y que fueron sometidos a reperfusión, el tratamiento con nitrato no produjo efectos sobre el área infartada, fracción eyectada, ni en el nivel plasmático de enzimas cardiacas (84). Por otra parte, en un estudio clínico reciente en pacientes con insuficiencia cardiaca (NCT01919177), el consumo de nitrato (12.9 mmol) contenido en jugo de betarragas, mejoró la respuesta al ejercicio (85). 
Función plaquetaria

Finalmente, otro posible blanco interesante para la salud cardiovascular de los nitritos y nitratos lo constituye la función plaquetaria. En las plaquetas, el NO también posee un rol regulador (5), inhibiendo su activación (86) y agregación, procesos cruciales en la formación de trombos (87). Por esto, los nitritos y nitratos plasmáticos potencialmente podrían contribuir a evitar la formación de trombos. Esto se ha descrito principalmente en el contexto de hipoxia celular, en donde la deoxihemoglobina contenida en los eritrocitos actuaria como nitrito reductasa, generando NO que inhibiría la agregación plaquetaria (88-91).

\section{CONCLUSIÓN}

Existe creciente evidencia de que el nitrato puede ejercer efectos benéficos en la salud, particularmente en el sistema cardiovascular, a través de su reducción a NO. Los vegetales son una fuente dietética importante de nitratos, pero se encuentran además como preservantes de alimentos cárneos. Tradicionalmente ha existido la visión de que los nitritos provenientes de esta última fuente son potencialmente cancerígenos, a través de la formación de nitrosaminas. Sin embargo no existe aún evidencia inequívoca que los nitratos sean responsables de ciertas formas de cáncer. Esto último es materia de actual debate.

En estudios clínicos, se ha verificado que el suplemento de la dieta con alimentos ricos en nitratos produce una reducción de la presión arterial y una mejora en la función vascular, pero aun no queda claro su efecto, al menos directo, sobre patologías cardíacas. Sea como fuere, los nitratos probablemente contribuyan a los efectos benéficos sobre la salud que se observa en las dietas con altos contenidos de vegetales.

\section{RESUMEN}

El consumo de vegetales está asociado a un menor riesgo de desarrollar enfermedades cardiovasculares y cáncer, efecto que tradicionalmente se le ha atribuido a su contenido en antioxidantes. Recientemente se ha propuesto que parte de estos efectos se deberían al contenido de nitratos en los vegetales. La enfermedades cardiovasculares se asocian a una disminución en la producción del óxido nítrico (NO). Una estrategia para corregir esta producción defectuosa es generar $\mathrm{NO}$ a partir de nitratos inorgánicos contenidos en los alimentos. Sin embargo, se ha propuesto que los nitratos, como los usados en el proceso de curación de carnes y preservación de alimentos, podrían generar aductos cancerígenos. Este paradigma está siendo revisado y la evidencia que los alimentos ricos en nitratos pudieran tener efectos benéficos sobre la salud, particularmente sobre parámetros cardiovasculares, ha generado resultados alentadores, aunque aun se necesitan estudio clínicos más completos.

Palabras clave: nitrito, nitrato, óxido nítrico, nitrosaminas, hipertensión arterial, nitrosilación.

\section{BIBLIOGRAFÍA}

1. Furchgott RF, Zawadzki JV. The obligatory role of endothelial cells in the relaxation of arterial smooth muscle by acetylcholine. Nature 1980;288:373-6.

2. Treuer AV, Gonzalez DR. Nitric oxide synthases, Snitrosylation and cardiovascular health: From molecular mechanisms to therapeutic opportunities (Review). Mol Med Rep. 2015;11:1555-65.

3. Gonzalez DR, Treuer A, Sun QA, Stamler JS, Hare JM. $S$-Nitrosylation of cardiac ion channels. J Cardiovasc Phar- macol. 2009;54:188-95.

4. Hare JM, Stamler JS. NO/redox disequilibrium in the failing heart and cardiovascular system. J Clin Invest. 2005;115:509-17.

5. Loscalzo J. Nitric oxide insufficiency, platelet activation, and arterial thrombosis. Circ Res. 2001;88:756-62.

6. Duran WN, Breslin JW, Sanchez FA. The NO cascade, eNOS location, and microvascular permeability. Cardiovasc Res. 2010;87:254-61.

7. Duran WN, Beuve AV, Sanchez FA. Nitric oxide, Snitrosation, and endothelial permeability. IUBMB Life 2013;65:819-26.

8. Cavieres V, Valdes $K$, Moreno B, Moore-Carrasco $R$, Gonzalez DR. Vascular hypercontractility and endothelial dysfunction before development of atherosclerosis in moderate dyslipidemia: role for nitric oxide and interleukin-6. Am J Cardiovasc Dis. 2014;4:114-22.

9. Giraldez RR, Panda A, Xia Y, Sanders SP, Zweier JL. Decreased nitric-oxide synthase activity causes impaired endothelium-dependent relaxation in the postischemic heart. J Biol Chem. 1997;272:21420-6.

10. Heiss C, Lauer T, Dejam A et al. Plasma nitroso compounds are decreased in patients with endothelial dysfunction. J Am Coll Cardiol. 2006;47:573-9.

11. Appel LJ, Moore TJ, Obarzanek E et al. A clinical trial of the effects of dietary patterns on blood pressure. DASH Collaborative Research Group. N Eng/ J Med. 1997;336:1117-24.

12. Joshipura KJ, Hu FB, Manson JE et al. The effect of fruit and vegetable intake on risk for coronary heart disease. Ann Intern Med. 2001;134:1106-14.

13. Knekt $P$, Jarvinen $R$, Dich J, Hakulinen T. Risk of colorectal and other gastro-intestinal cancers after exposure to nitrate, nitrite and N-nitroso compounds: a follow-up study. Int J Cancer 1999;80:852-6.

14. Kuhnle GG, Story GW, Reda T et al. Diet-induced endogenous formation of nitroso compounds in the GI tract. Free Radic Biol Med. 2007;43:1040-7.

15. Avasilcai L, Cuciureanu R. [Nitrates and nitrites in meat products--nitrosamines precursors]. Rev Med Chir Soc Med Nat Lasi. 2011;115:606-11.

16. Mirvish SS, Haorah J, Zhou L, Clapper ML, Harrison $K L$, Povey AC. Total N-nitroso compounds and their precursors in hot dogs and in the gastrointestinal tract and feces of rats and mice: possible etiologic agents for colon cancer. $J$ Nutr. 2002;132:3526S-9S.

17. van Loon AJ, Botterweck AA, Goldbohm RA, Brants $H A$, van Klaveren $J D$, van den Brandt PA. Intake of nitrate and nitrite and the risk of gastric cancer: a prospective cohort study. Br J Cancer 1998;78:129-35.

18. Lundberg JO, Weitzberg E, Cole JA, Benjamin N. Nitrate, bacteria and human health. Nat Rev Microbiol. 2004;2:593-602.

19. Benjamin $N, O^{\prime}$ Driscoll $F$, Dougall $H$ et al. Stomach NO synthesis. Nature 1994;368:502.

20. Cosby K, Partovi KS, Crawford JH et al. Nitrite reduction to nitric oxide by deoxyhemoglobin vasodilates the human circulation. Nat Med. 2003;9:1498-505.

21. Jansson EA, Huang $L$, Malkey $R$ et al. A mammalian functional nitrate reductase that regulates nitrite and nitric oxide homeostasis. Nat Chem Biol. 2008;4:411-7.

22. Webb A, Bond R, McLean P, Uppal R, Benjamin N, Ahluwalia $A$. Reduction of nitrite to nitric oxide during ischemia protects against myocardial ischemia-reperfusion damage. Proc Natl Acad Sci. U S A 2004;101:13683-8. 
23. Duranski MR, Greer JJ, Dejam A et al. Cytoprotective effects of nitrite during in vivo ischemia-reperfusion of the heart and liver. J Clin Invest. 2005;115:1232-40.

24. Tripatara $P$, Patel NS, Webb A et al. Nitrite-derived nitric oxide protects the rat kidney against ischemia/reperfusion injury in vivo: role for xanthine oxidoreductase. J Am Soc Nephrol. 2007;18:570-80.

25. Jung $K H, C h u K$, Lee ST et al. Augmentation of nitrite therapy in cerebral ischemia by NMDA receptor inhibition. Biochem Biophys Res Commun. 2009;378:507-12.

26. Jung $K H$, Chu $K$, Lee ST et al. Effects of long term nitrite therapy on functional recovery in experimental ischemia model. Biochem Biophys Res Commun. 2010;403:66-72.

27. Carlstrom M, Larsen FJ, Nystrom T et al. Dietary inorganic nitrate reverses features of metabolic syndrome in endothelial nitric oxide synthase-deficient mice. Proc Natl Acad Sci. U S A. 2010;107:17716-20.

28. Weitzberg E, Lundberg JO. Novel aspects of dietary nitrate and human health. Annu Rev Nutr. 2013;33:129-59.

29. Leaf CD, WishnokJS, Tannenbaum SR. L-arginine is a precursor for nitrate biosynthesis in humans. Biochem Biophys Res Commun. 1989;163:1032-7.

30. Bondonno CP, Liu AH, Croft KD et al. Short-term effects of nitrate-rich green leafy vegetables on blood pressure and arterial stiffness in individuals with high-normal blood pressure. Free Radic Biol Med. 2014;77:353-62.

31. Kapil V, Khambata RS, Robertson A, Caulfield MJ, Ahluwalia A. Dietary nitrate provides sustained blood pressure lowering in hypertensive patients: a randomized, phase 2, double-blind, placebo-controlled study. Hypertension 2015;65:320-7.

32. Spiegelhalder B, Eisenbrand $G$, Preussmann R. Influence of dietary nitrate on nitrite content of human saliva: possible relevance to in vivo formation of $\mathrm{N}$-nitroso compounds. Food Cosmet Toxicol. 1976;14:545-8.

33. Potter L, Angove H, Richardson D, Cole J. Nitrate reduction in the periplasm of gram-negative bacteria. Adv Microb Physiol. 2001;45:51-112.

34. Richardson DJ, Berks BC, Russell DA, Spiro S, Taylor CJ. Functional, biochemical and genetic diversity of prokaryotic nitrate reductases. Cell Mol Life Sci. 2001;58:165-78.

35. Archer MC. Mechanisms of action of N-nitroso compounds. Cancer Surv. 1989;8:241-50.

36. Lundberg JO, Weitzberg E, Gladwin MT. The nitrate-nitritenitric oxide pathway in physiology and therapeutics. Nat Rev Drug Discov. 2008;7:156-67.

37. Kozlov AV, Staniek K, Nohl H. Nitrite reductase activity is a novel function of mammalian mitochondria. FEBS Lett. 1999;454:127-30.

38. Huang Z, Shiva S, Kim-Shapiro DB et al. Enzymatic function of hemoglobin as a nitrite reductase that produces NO under allosteric control. J Clin Invest. 2005; 115: 2099-107.

39. Honikel $\mathrm{KO}$. The use and control of nitrate and nitrite for the processing of meat products. Meat Sci. 2008;78:68-76.

40. Jakszyn P, Gonzalez CA. Nitrosamine and related food intake and gastric and oesophageal cancer risk: a systematic review of the epidemiological evidence. World J Gastroenterol. 2006;12:4296-303.

41. Jakszyn P, Bingham S, Pera $G$ et al. Endogenous versus exogenous exposure to $\mathrm{N}$-nitroso compounds and gastric cancer risk in the European Prospective Investigation into Cancer and Nutrition (EPIC-EURGAST) study. Carcinogenesis 2006;27:1497-501.
42. Bryan NS, Alexander DD, Coughlin JR, Milkowski AL, Boffetta $P$. Ingested nitrate and nitrite and stomach cancer risk: an updated review. Food Chem Toxicol. 2012;50:3646-65.

43. Yang CY, Hung CF. Colon cancer mortality and total hardness levels in Taiwan's drinking water. Arch Environ Contam Toxicol. 1998;35:148-51.

44. Tannenbaum SR, Wishnok JS, Leaf CD. Inhibition of nitrosamine formation by ascorbic acid. Am J Clin Nutr. 1991;53:247S-50S.

45. Licht WR, Tannenbaum SR, Deen WM. Use of ascorbic acid to inhibit nitrosation: kinetic and mass transfer considerations for an in vitro system. Carcinogenesis 1988;9:365-72.

46. Licht WR, Fox JG, Deen WM. Effects of ascorbic acid and thiocyanate on nitrosation of proline in the dog stomach. Carcinogenesis 1988;9:373-7.

47. Kujawska M, Ignatowicz E, Murias M, Ewertowska $M$, Mikolajczyk K, Jodynis-Liebert J. Protective effect of red beetroot against carbon tetrachloride- and N-nitrosodiethylamine-induced oxidative stress in rats. J Agric Food Chem. 2009;57:2570-5.

48. Milkowski A, Garg HK, Coughlin JR, Bryan NS. Nutritional epidemiology in the context of nitric oxide biology: a riskbenefit evaluation for dietary nitrite and nitrate. Nitric Oxide 2010;22:110-9.

49. Larauche M, Bueno L, Fioramonti J. Effect of dietary nitric oxide on gastric mucosal mast cells in absence or presence of an experimental gastritis in rats. Life Sci. 2003;73:1505-16.

50. Larauche M, Anton PM, Garcia-Villar $R$ et al. Protective effect of dietary nitrate on experimental gastritis in rats. Br J Nutr. 2003;89:777-86.

51. Bjorne HH, Petersson J, Phillipson M, Weitzberg E, Holm L, Lundberg JO. Nitrite in saliva increases gastric mucosal blood flow and mucus thickness. J Clin Invest. 2004;113:106-14.

52. Brown JF, Hanson PJ, Whittle BJ. Nitric oxide donors increase mucus gel thickness in rat stomach. Eur J Pharmacol. 1992;223:103-4.

53. Stein $L Y, K$ lotz MG. Nitrifying and denitrifying pathways of methanotrophic bacteria. Biochem Soc Trans. 2011;39:1826-31.

54. Lidder S, Webb AJ. Vascular effects of dietary nitrate (as found in green leafy vegetables and beetroot) via the nitrate-nitrite-nitric oxide pathway. Br J Clin Pharmacol. 2013;75:677-96.

55. Kujala TS, Loponen JM, Klika KD, Pihlaja K. Phenolics and betacyanins in red beetroot (Beta vulgaris) root: distribution and effect of cold storage on the content of total phenolics and three individual compounds. J Agric Food Chem. 2000;48:5338-42.

56. Palomo I, Gutierrez M, Astudillo L et al. Efecto Antioxidante de Frutas y Hortalizas de la Zona Central de Chile. Rev Chil Nutr. 2009;36:152-8.

57. Santamaria P, Elia A, Serio F, Todaro F. A survey of nitrate and oxalate content in fresh vegetables. J Sci Food Agric. 1999;79:1882-8.

58. Santamaria P. Nitrate in vegetables: toxicity, content, intake and EC regulation. J Sci Food Agric. 2006;86:10-7.

59. Carrasco G, Rebolledo P, Urrestarazu M, Tapia J. Contenido de nitratos en lechugas tipo mantecosas cultivadas en diferentes sistemas en la zona centro - sur de Chile. Información Tecnológica 2001;12:51-4.

60. Carrasco G, Tapia J, Urrestarazu M. Contenido de Nitratos en Lechugas Cultivadas en Sistemas Hidroponicos. Idesia 
2006:24:25-30.

61. Paillan H, Cuevas N, Carrasco G. Nitrate and ascorbic acid contents of the radish (Raphanus sativus L.) under organic greenhouse conditions. Phyton 2001 (Special Issue):141-6.

62. Dejam A, Hunter CJ, Tremonti C et al. Nitrite infusion in humans and nonhuman primates: endocrine effects, pharmacokinetics, and tolerance formation. Circulation 2007;116:1821-31.

63. Kapil V, Weitzberg E, Lundberg JO, Ahluwalia A. Clinical evidence demonstrating the utility of inorganic nitrate in cardiovascular health. Nitric Oxide 2014;38:45-57.

64. Webb AJ, Patel N, Loukogeorgakis S et al. Acute blood pressure lowering, vasoprotective, and antiplatelet properties of dietary nitrate via bioconversion to nitrite. Hypertension 2008:51:784-90.

65. Zielinska-Przyjemska M, Olejnik A, Dobrowolska-Zachwieja $A$, Grajek W. In vitro effects of beetroot juice and chips on oxidative metabolism and apoptosis in neutrophils from obese individuals. Phytother Res. 2009;23:49-55.

66. Larsen FJ, Ekblom B, Sahlin K, Lundberg JO, Weitzberg E. Effects of dietary nitrate on blood pressure in healthy volunteers. N Engl J Med. 2006;355:2792-3.

67. Coles LT, Clifton PM. Effect of beetroot juice on lowering blood pressure in free-living, disease-free adults: a randomized, placebo-controlled trial. Nutr J. 2012;11:106.

68. Hobbs DA, Kaffa N, George TW, Methven L, Lovegrove JA. Blood pressure-lowering effects of beetroot juice and novel beetroot-enriched bread products in normotensive male subjects. Br J Nutr. 2012;108:2066-74.

69. Kapil V, Milsom AB, Okorie $M$ et al. Inorganic nitrate supplementation lowers blood pressure in humans: role for nitrite-derived NO. Hypertension 2010;56:274-81.

70. Sobko T, Marcus C, Govoni M, Kamiya S. Dietary nitrate in Japanese traditional foods lowers diastolic blood pressure in healthy volunteers. Nitric Oxide 2010;22:136-40.

71. Bondonno $C P$, Yang $X$, Croft $K D$ et al. Flavonoid-rich apples and nitrate-rich spinach augment nitric oxide status and improve endothelial function in healthy men and women: a randomized controlled trial. Free Radic Biol Med. 2012;52:95-102.

72. Hobbs DA, Goulding MG, Nguyen A et al. Acute ingestion of beetroot bread increases endothelium-independent vasodilation and lowers diastolic blood pressure in healthy men: a randomized controlled trial. J Nutr. 2013;143:1399-405.

73. Jajja A, Sutyarjoko A, Lara J et al. Beetroot supplementation lowers daily systolic blood pressure in older, overweight subjects. Nutr Res. 2014;34:868-75.

74. Gilchrist $M$, Winyard PG, Fulford J, Anning C, Shore AC, Benjamin N. Dietary nitrate supplementation improves reaction time in type 2 diabetes: Development and application of a novel nitrate-depleted beetroot juice placebo. Nitric Oxide 2014;40C:67-74.

75. Siervo M, Lara J, Ogbonmwan I, Mathers JC. Inorganic nitrate and beetroot juice supplementation reduces blood pressure in adults: a systematic review and meta-analysis. J Nutr. 2013;143:818-26.

76. Rammos C, Hendgen-Cotta UB, Sobierajski J, Bernard
A, Kelm M, Rassaf T. Dietary nitrate reverses vascular dysfunction in older adults with moderately increased cardiovascular risk. J Am Coll Cardiol. 2014;63:1584-5.

77. Presley TD, Morgan AR, Bechtold E et al. Acute effect of a high nitrate diet on brain perfusion in older adults. Nitric Oxide 2011;24:34-42.

78. Simon JN, Duglan D, Casadei B, Carnicer R. Nitric oxide synthase regulation of cardiac excitation-contraction coupling in health and disease. J Mol Cell Cardiol. 2014;73:80-91.

79. Bhushan S, Kondo K, Polhemus DJ et al. Nitrite therapy improves left ventricular function during heart failure via restoration of nitric oxide-mediated cytoprotective signaling. Circ Res. 2014;114:1281-91.

80. Carlstrom M, Persson AE, Larsson $E$ et al. Dietary nitrate attenuates oxidative stress, prevents cardiac and renal injuries, and reduces blood pressure in salt-induced hypertension. Cardiovasc Res. 2011;89:574-85.

81. Rassaf T, Totzeck M, Hendgen-Cotta UB, Shiva S, Heusch $G$, Kelm M. Circulating nitrite contributes to cardioprotection by remote ischemic preconditioning. Circ Res. 2014;114:1601-10.

82. Shiva S, Sack MN, Greer JJ et al. Nitrite augments tolerance to ischemia/reperfusion injury via the modulation of mitochondrial electron transfer. J Exp Med. 2007;204:2089102.

83. Ingram TE, Fraser AG, Bleasdale RA et al. Low-dose sodium nitrite attenuates myocardial ischemia and vascular ischemia-reperfusion injury in human models. J Am Coll Cardiol. 2013;61:2534-41.

84. Siddiqi N, Neil C, Bruce $M$ et al. Intravenous sodium nitrite in acute ST-elevation myocardial infarction: a randomized controlled trial (NIAMI). Eur Heart J. 2014;35:1255-62.

85. Zamani $P$, Rawat $D$, Shiva-Kumar $P$ et al. Effect of inorganic nitrate on exercise capacity in heart failure with preserved ejection fraction. Circulation 2015;131:371-80.

86. Morrell CN, Matsushita $K$, Chiles $K$ et al. Regulation of platelet granule exocytosis by S-nitrosylation. Proc Natl Acad Sci U S A. 2005;102:3782-7.

87. Palomo I, Fuentes E, Padro T, Badimon L. Platelets and atherogenesis: Platelet anti-aggregation activity and endothelial protection from tomatoes (Solanum lycopersicum L.). Exp Ther Med. 2012;3:577-84.

88. Apostoli GL, Solomon A, Smallwood MJ, Winyard PG, Emerson $M$. Role of inorganic nitrate and nitrite in driving nitric oxide-cGMP-mediated inhibition of platelet aggregation in vitro and in vivo. J Thromb Haemost. 2014;12:1880-9.

89. Park JW, Piknova B, Huang PL, Noguchi CT, Schechter AN. Effect of blood nitrite and nitrate levels on murine platelet function. PLoS One 2013;8:e55699.

90. Srihirun S, Sriwantana T, Unchern Set al. Platelet inhibition by nitrite is dependent on erythrocytes and deoxygenation. PLoS One 2012;7:e30380.

91. Velmurugan S, Kapil V, Ghosh SM et al. Antiplatelet effects of dietary nitrate in healthy volunteers: Involvement of CGMP and influence of sex. Free Radic Biol Med. 2013. 